\title{
Inflammatory Cytokines Response to Isometric Handgrip Exercise and the Effects of Duration and Intensity of the Isometric Effort in Prehypertensive Subjects
}

GODSDAY UDOJI OGBUTOR ( $\sim$ godsday.ogbutor@fmcasaba.org )

Federal Medical CENTRE ASABA https://orcid.org/0000-0002-0476-4492

Eze Kingsley Nwangwa

DSU: Delta State University

Collins Ogbeivor

Al Mashfa International Hospital, Riyadh

Nkemakonam Ezeonu

Federal Medical Centre Asaba

Ephraim Chukwuemeka

Federal Medical Centre, Asaba

Chinedu Francis Ugoeze

Federal Medical Centre, Asaba

Emmanuel Ezunu

Federal Medical Centre, Asaba

Zuwaira Odegua Nwabueze

Federal Medical Centre, Asaba

Igweh Chukwuka John

Delta State University, Abraka

Research

Keywords: Chronic diseases, Subjects, Inflammatory Cytokines, Handgrip, Exercise, Pre-hypertensive

Posted Date: January 3rd, 2022

DOI: https://doi.org/10.21203/rs.3.rs-1125185/v1

License: (c) (i) This work is licensed under a Creative Commons Attribution 4.0 International License. Read Full License 


\section{Abstract}

\section{BACKGROUND}

Chronic low grade systemic inflammation has been identified as a major risk factor for chronic diseases. The potential for physical exercise to induce anti-inflammatory effect is now increasingly being explored but there is paucity of data regarding the effects isometric exercise on inflammatory cytokines. The objective of this study was to investigate the responses of selected inflammatory cytokines to isometric handgrip exercise and identify possible effects of intensity and duration of the isometric effort on these variables. CASE PRESENTATION: A total of one hundred and ninety two $(\mathrm{N}=192)$ sedentary prehypertensive subjects, aged between 30-50years were recruited into the study and randomly distributed into three groups of 64 subjects each. The subjects performed a 24 consecutive day's isometric hand grip exercise at $30 \%$ Maximum Voluntary Contraction. At the end of the 24 days, the group one (GP1) discontinued with the exercise protocol while the group two (GP2) continued the exercise protocol for another 24 consecutive days and the group three (GP3) continued with the exercise protocol for another 24 consecutive days but at 50\%MVC. The parameters used to assess for the inflammatory cytokine variables included interleukin 10, interleukin 6 and tumor necrotic factor. At the end of the study, there was an increase in the resting values of interleukin 10 across the three groups while the resting values of interleukin 6 and tumor necrotic factor reduced significantly across groups. CONCLUSIONS: The reductions noted in the pro-inflammatory cytokines and increase in the anti-inflammatory cytokines could have a positive impact in the management of chronic diseases. It was also found that increase in intensity and/or duration produced more proportionate effect.

\section{Introduction}

Systemic inflammation has been identified as a major underlying predisposing factor for several chronic diseases [1, 2, 3, and 4]. According to Beavers et al., elevated serum concentration of pro-inflammatory cytokines are related with pathological conditions of several systems and organs of the body [4]. The prevalence of chronic disease has increased steadily in recent years that nearly half of the population of the world suffers from at least one chronic condition, and the number is growing [5]. The Centers for Disease Control and Prevention states that over $60 \%$ of all deaths are attributable to an underlying chronic disease. The scientific community has further projected that by 2030 , chronic diseases will result in over $75 \%$ of global deaths [6]. The beginning of the 21 st century witnessed the upsurge of chronic diseases and are essentially related to behavioural patterns, and can be curtailed or prevented by lifestyle modifications [7].

Due to the increasing longevity and growing aging population and a global increase in risk factors for chronic diseases, alternative interventions are required to minimize the global and economic burden of chronic disease. Considering the wide variety and range of affectation and prevalence of chronic diseases, a population health approach, by means of preventive measures, would be the most appropriate model to adopt in dealing with this ubiquitous problem. Such preventative measures would 
help in reducing the global burden of chronic diseases plaguing the health care systems of the world [2]. Regular physical exercise has been reported to create an anti-inflammatory milieu leading to reduced resting levels of pro-inflammatory cytokines and increased levels of anti-inflammatory cytokine concentrations in both younger and older adults $[8,9]$.

Physical exercise is recognized as an important strategy for reducing the risk of chronic disease with contemporary studies focusing on the role of physical exercise in the management of the inflammatory profile. Physical exercise has equally been shown to symbolize a measureable model of stress found to induce a pattern of hormonal and immunological responses [10]. This pattern of response is categorized as a physiological defense response, with the primary objective of stimulating a healing process. The magnitude of this process is the determinant of the health benefit and is primarily regulated by the concentration of pro- and anti-inflammatory cytokines. Localized and short term inflammatory response is believed to be physiologically beneficial and protective to an acute tissue injury but a prolonged or chronic response resulting from elevated levels of pro-inflammatory cytokines could lead to Systemic inflammatory response syndrome (SIRS) which is unhealthy and could lead to death [11]. The overproduction of pro-inflammatory cytokines leads to this syndrome and could result in multiple organ damage, associated with thermal and ischemia-reperfusion injury, severe trauma, septic shock and systemic infections $[12,13]$.

Over the last century, sedentary lifestyle is increasingly becoming a norm with more people becoming less active, thus, this has been promoted either by the alteration in the kind of work, or by adoption of new habits attributable in part to changes in the demands of work. This scenario has resulted in an unexpected increase in the prevalence of chronic diseases [14]. Physical exercise is considered an effective and preventive countermeasure to chronic diseases and findings from previous studies indicated that physical exercise improves many components of cardiovascular risk factor as well as insulin sensitivity [15]. Unfortunately, physical activity is becoming increasingly difficult to sustain. This is because the society is advancing rapidly in technology that most people today are spared the burden of physical labour, coupled with the fact that most occupations do not require significant physical activity. Nowadays, leisure time is even filled with sedentary behaviors [16].

Physical exercise recommendations have centered on dynamic exercise and there has been enormous restrictions and caution with regard to the recommendations of isometric exercise. Chrysant et al. observed that there is paucity of data regarding recommendations for isometric or resistance exercise [17]. Despite much evidence indicating that increased physical activity is associated with reduced systemic inflammation, the results of intervention studies are still controversial. One promising regimen, currently being explored, is isometric hand grip exercise training [17]. Preliminary studies have shown that this type of exercise may have a positive impact on inflammatory profile. This study is therefore designed to provide evidence for this time-efficient and cost-effective therapy. Moreover, handgrip exercise is easy to perform and may be accomplished in various locations such as hospitals, schools, in transit and in the home, enabling increased adherence to treatment, in contrast with other forms of exercises. 


\section{Materials And Method}

\section{General Study Design}

The procedure of this study consisted of an isometric handgrip exercise trial. One hundred and ninety two prehypertensives $(n=192$, males $=105$ and females $=87$, age, 39.04 \pm 6.4 years; body mass index, $25.45 \pm 2.72 \mathrm{~kg} / \mathrm{m}^{2}$ ), were recruited for the study. All the subject were diagnosed and referred by the physician with a blood pressure level classified as prehypertension based on the classification of the Joint National Committee on Prevention, Detection, Evaluation, and Treatment of High Blood Pressure. This represents a systolic blood pressure range of $120-139 \mathrm{mmHg}$ and diastolic blood pressure range of $80-89 \mathrm{mmHg}$. The subjects had an age ranging from 30 to $50 y e a r s$. This is because inflammatory diseases and prehypertension have been found to have an increased risk in individuals 40years and above.

A screening session was conducted to assess the baseline parameters and blood pressure of the subjects and blood samples were collected. All blood sample collection and blood pressure measurements were done according to international guidelines. The sample population was randomly selected into any of the three groups. The subjects were asked to pick from a ballot box, concealed papers marked G1, G2 or G3 to determine which group the subjects should belong. A detailed procedure of the exercise was then given to the subjects before commencement of the exercise training.

\section{Inclusion criteria}

Inclusion into the study was subject to a normal medical examination, determined by a consultant Physician. Only subjects, with no clinical evidence of infectious, inflammatory and/or chronic diseases were recruited. More so, subjects were not on medication and all the participants recruited were sedentary which was defined by a score of three or less using the Rapid Assessment of Physical Activity survey. The subjects were properly briefed and written informed consent was obtained. This study conformed to the Declaration of Helsinki and jointly received institutional ethical approval of Federal Medical Centre Asaba, Delta State (FMC/ASB/A81.VOLXII/101) and Faculty of Basic Medical Sciences, Delta State University, Abraka, Delta State (REC/FBMS/DELSU/18/16/103).

\section{Exclusion Criteria}

Subjects were excluded from the study if their age is below and or above the age range of 30-50years or have a queried health status with clinical evidence of chronic diseases, a blood pressure above or below the prehypertension level, on medications and/or subjects who declined to participate in the study. Other exclusion criteria for Isometric Hand Grip include individuals suffering from debilitating arthritis, carpel tunnel, peripheral neuropathy, an aneurysm, or mitral valve complications.

\section{Experimental Procedure}


On arrival at the clinic on the first day, subjects were made to observe a 15 minutes seated rest after which their blood samples were collected for baseline levels of inflammatory cytokine (TNF-a, IL6 and IL10). A detailed explanation and a demonstration of the exercise protocol were given to the subjects and they were asked to report at the Physiotherapy clinic at $4.00 \mathrm{pm}$, for the exercise daily. The training session for each day took place between the hours of $4.00 \mathrm{pm}$ and $8.00 \mathrm{pm}$ daily.

The subjects on arrival at the clinic were made to observe a 15 minutes seated rest after which they were asked to squeeze the dynamometer with their dominant hand twice, for a maximum of 2 seconds with a five minutes rest in between; so as to determine their respective maximum voluntary contraction (M.V.C) for each session. The mean of the two readings was taken as the MVC for each subject for that session. Subjects were thereafter instructed to squeeze and sustain the dynamometer for 2 minutes at $30 \%$ M.V.C. The dynamometer pointer which read the scale gave a visual feedback to the subjects for the maintenance of the $30 \%$ M.V.C.

This procedure was repeated twice for each training session with a 5 minutes rest in between. The position adopted by the subjects throughout the exercise training was sitting with upper limbs supported on a table. The exercise protocol was done for 24 consecutive days. The group one (G1) discontinued with the exercise protocol after 24 days while the group two (G2) continued for another 24 consecutive days at $30 \%$ MVC. On the other hand, the group three (G3) continued with the exercise protocol for another 24 consecutive days but at $50 \% \mathrm{MVC}$. At the end of the 48 days, blood samples were collected again on the $49^{\text {th }}$ day for assessment of the resting data of the inflammatory cytokines (TNFa, IL6 and IL10) parameters.

\section{Blood Sample Collection and Analysis of Cytokines}

Five milliliters of overnight fasting (10-12hr) blood samples were intravenously collected pre- and postexercise. The pre-exercise blood sample collection was done on the on the first day of the screening prior to the commencement of the exercise between the hours of 7-9am and the post exercise blood sample was collected on the $49^{\text {th }}$ day to the commencement of the exercise at the same time. After $10 \mathrm{~min}$ of resting in a chair, venous blood from the ante cubital vein was collected into a serum tube. Immediately following collection, blood samples were allowed to settle at room temperature for 20 minutes to 1 hour in the vacutainer tubes using standard aseptic techniques to be clotted, and then was centrifuged $(1,000$ g) at $4^{\circ} \mathrm{C}$ for $20 \mathrm{~min}$ to separate serum from plasma.

Serum samples were then allocated into $1.5 \mathrm{~mL}$ tubes and immediately frozen for further analyses. Commercially available ELISA kits were then used to measure interleukin 6 (IL-6) interleukin 10 (IL-10) and Tumor necrotic factor (TNF-a) concentrations. Samples were analyzed in duplicate and all techniques and materials were used according to the manufacturer's instructions by qualified and licensed medical laboratory scientists. All samples for any one participant were analyzed using the same assay to eliminate inter-assay variance. 
Three major inflammatory cytokines were selected based on their peculiar role/characteristics and ease of laboratory assay with regard to available resources and facilities. These are interleukin 6 (IL-6) interleukin 10 (IL-10) and Tumor necrotic factor (TNF-a). which is a major pro-inflammatory cytokine and has been shown to modulate multiple signaling pathways with wide-ranging down streams effect $[18,19]$. TNF-a plays a vital role in the typical immune response, through the modulation of pathways that involve an immediate inflammatory response with subsequent proliferation and programmed cell death. It has been fingered in a number of inflammatory diseases, particularly in rheumatoid arthritis, ankylosing spondylitis, and chrohn's disease [19] while IL-6 exert pro-inflammatory effect when the signaling receptor protein which activates the membrane-bound signaling receptor protein, thus, higher levels of cardiopulmonary fitness have been associated with lower circulating concentrations of both IL- 6 and CRP at rest $[1,2,9]$.

The collected data were statistically analyzed using the descriptive and inferential statistics. The descriptive statistics employed in this study were the mean and standard deviation. The inferential statistics used in the analysis of the data included a one tailed student's t-Test to determine the intragroups differences in the initial and final values of the parameters, of the three groups. One way analysis of variance was thereafter used to compare the means of the three groups to determine their level of relationship. Furthermore, a two tailed student's t-Test was used to determine the intra-groups differences in the initial and final resting values of the parameters of group one and group two; and group two and group three to determine the effect of continuation (Duration) and increase in dosage (Intensity) of the isometric effort respectively.

\section{Results}

The results are presented in tables and figures.

Table 1

Descriptive Demographic Data of the Participants

\begin{tabular}{|llll|}
\hline Variables & N & Mean & Std. Deviation \\
\hline Age (years) & 192 & 39.04 & 6.441 \\
\hline Height (M) & 192 & 1.7000 & .11299 \\
\hline Weight (kg) & 192 & 73.3750 & 9.00975 \\
\hline BMl (kg/m2) & 192 & 25.4487 & 2.72359 \\
\hline
\end{tabular}

Table 1 above is a descriptive demographic data of all the participants. A total of one hundred and ninety two subjects with a mean age of $39.04 \pm 6.4$ years, height $1.7 \pm 0.11 \mathrm{~m}$, weight $73.4 \pm 9.0 \mathrm{~kg}$ and Body mass index of $25.4 \pm 2.7 \mathrm{~kg} / \mathrm{m}^{2}$

Figure 1 represents a pie chart showing the BMI distribution of the participants. The chart shows that $3.1 \%, 15.6 \%, 34.4 \%$ and $46.9 \%$ of the subjects were morbidly obese, obese, overweight and normal with 
their weight respectively. This shows that six (6) people were morbidly obese, 30 participants were obese, sixty six participants overweight and 90 participants had normal weight. From this data weight maybe related to prehypertension since the participants were randomly selected though this was not the focus of the study

Figure 2 above represents pie chart showing the distribution of the participants based on their sex. A total of $54.7 \%$ (105) were males while the rest $45.3 \%$ (87) were females. It could be deduced from this data that prehypertension is more associated with males.

Table 2

Pre and Post Exercise mean values of the Inflammatory Cytokines of exercise group 1

\begin{tabular}{|lllllll|}
\hline Parameters & Pre-Exercise & Post-Exercise & Diff. & Df & Sig.(2-tailed) & Remark \\
\hline TNF- $\mathrm{a}(\mathrm{pg} / \mathrm{ml})$ & $1.63 \pm 0.26$ & $1.58 \pm 0.26$ & $-0.05 \pm 0.04$ & 63 & $<0.001^{*}$ & Significant \\
\hline IL-6(pg/ml) & $0.45 \pm 0.08$ & $0.42 \pm 0.08$ & $-0.04 \pm 0.01$ & 63 & $<0.001^{*}$ & Significant \\
\hline IL- $10(\mathrm{pg} / \mathrm{ml})$ & $6.5 \pm 1.58$ & $7.03 \pm 1.59$ & $0.53 \pm 0.14$ & 63 & $<0.001^{*}$ & Significant \\
\hline
\end{tabular}

\section{Values are expressed as mean \pm Standard Deviation (S.D), $n=64 . * P<0.05$}

Table 2 above shows the pre and post exercise mean values of the inflammatory cytokines of the exercise group 1. Results obtained revealed a mean reduction of $0.05 \pm 0.036 \mathrm{pg} / \mathrm{ml}$ and $0.036 \pm 0.017 \mathrm{pg} / \mathrm{ml}$ in the tumor necrotic factor (TNF-a) and interleukin 6 (IL-6) respectively and a mean increase of $0.53 \pm 0.14 \mathrm{pg} / \mathrm{ml}$ in interleukin 10 (IL-10). These values were statistically significant. This means that the exercise protocol of group 1 produced a significant mean reduction in the values of tumor necrotic factor (TNF-a) and interleukin 6 (IL-6) and a significant mean increase of in interleukin 10 (IL-10). It appears then that isometric handgrip exercise at $30 \% \mathrm{MVC}$ has anti-inflammatory effect in humans

Table 3

Pre and Post Exercise mean values of the Inflammatory Cytokines of exercise group 2

\begin{tabular}{|lllllll|}
\hline Parameters & Pre-Exercise & Post-Exercise & Diff. & Df & Sig.(2-tailed) & Remark \\
\hline TNF-a $(\mathrm{pg} / \mathrm{ml})$ & $1.47 \pm 0.27$ & $1.41 \pm 0.26$ & $-0.07 \pm 0.03$ & 63 & $<0.001^{*}$ & Significant \\
\hline $\mathrm{IL}-6(\mathrm{pg} / \mathrm{ml})$ & $0.47 \pm 0.10$ & $0.41 \pm 0.09$ & $-0.06 \pm 0.01$ & 63 & $<0.001 *$ & Significant \\
\hline $\mathrm{IL}-10(\mathrm{pg} / \mathrm{ml})$ & $6.2 \pm 1.3$ & $6.69 \pm 1.44$ & $0.49 \pm 0.24$ & 63 & $<0.001 *$ & Significant \\
\hline
\end{tabular}

\section{Values are expressed as mean \pm Standard Deviation (S.D), $\mathrm{n}=64 .{ }^{\mathrm{P}}<0.05$}

Table 3 above shows the pre and post exercise mean values of the inflammatory cytokines of the exercise group 2. The participants show a mean reduction of $0.065 \pm 0.026 \mathrm{pg} / \mathrm{ml}$ and $0.057 \pm 0.014 \mathrm{pg} / \mathrm{ml}$ 
in the tumor necrotic factor (TNF-a) and interleukin 6 (IL-6) respectively and an increase of $0.493 \pm 0.24 \mathrm{pg} / \mathrm{ml}$ in interleukin 10 (IL-10). The values were statistically significant. This means that isometric handgrip exercise at $30 \% \mathrm{MVC}$ over a longer duration also promote anti-inflammatory effect in humans

Table 4

Pre and Post Exercise mean values of the Inflammatory Cytokines of exercise group 3

\begin{tabular}{|lllllll|}
\hline Parameters & Pre-Exercise & Post-Exercise & Diff. & Df & Sig.(2-tailed) & Remark \\
\hline TNF- $\mathrm{a}(\mathrm{pg} / \mathrm{ml})$ & $1.52 \pm 0.16$ & $1.41 \pm 0.16$ & $-0.10 \pm 0.11$ & 63 & $0.003^{*}$ & Significant \\
\hline $\mathrm{IL}-6(\mathrm{pg} / \mathrm{ml})$ & $0.47 \pm 0.11$ & $0.40 \pm 0.10$ & $-0.07 \pm 0.28$ & 63 & $<0.001^{*}$ & Significant \\
\hline $\mathrm{IL}-10(\mathrm{pg} / \mathrm{ml})$ & $6.53 \pm 1.25$ & $7.31 \pm 1.30$ & $0.77 \pm 0.32$ & 63 & $<0.001^{*}$ & Significant \\
\hline
\end{tabular}

\section{Values are expressed as mean \pm Standard Deviation (S.D), $\mathrm{n}=64 .{ }^{*} \mathrm{P}<0.05$}

Table 4 above shows the Pre and Post Exercise mean values of the Inflammatory Cytokines of exercise group 3. The participants also had a mean reduction of $0.10 \pm 0.11 \mathrm{pg} / \mathrm{ml}$ and $0.07 \pm 0.28 \mathrm{pg} / \mathrm{ml}$ in tumor necrotic factor (TNF- $\alpha$ ) and interleukin 6 (IL-6) respectively and an increase of $0.77 \pm 0.32 \mathrm{pg} / \mathrm{ml}$ in interleukin 10 (IL-10). The values were statistically significant. This means that isometric handgrip exercise at $30 \% \mathrm{MVC}$ over a longer duration and at increased intensity also promote anti-inflammatory effect in humans

Table 5

The Comparative Effects of Cessation and Continuation of the Exercise Protocol at the End of 48days on Inflammatory Cytokines

\begin{tabular}{|lllllll|}
\hline Parameters & $\begin{array}{l}\text { Exercise } \\
\text { Group. I }\end{array}$ & $\begin{array}{l}\text { Exercise Group } \\
\mathbf{2}\end{array}$ & Diff. & Df & $\begin{array}{l}\text { Sig.(2- } \\
\text { tailed) }\end{array}$ & Remark \\
\hline $\begin{array}{l}\text { TNF- } \\
\mathrm{a}(\mathrm{pg} / \mathrm{ml})\end{array}$ & $0.05 \pm 0.036$ & $0.07 \pm 0.026$ & $0.015 \pm 0.01$ & 127 & 0.553 & Insignificant \\
\hline $\mathrm{IL}-6(\mathrm{pg} / \mathrm{ml})$ & $0.036 \pm 0.017$ & $0.06 \pm 0.014$ & $0.021 \pm 1.35$ & 127 & $0.001^{*}$ & Significant \\
\hline $\begin{array}{l}\mathrm{IL}- \\
10(\mathrm{pg} / \mathrm{ml})\end{array}$ & $0.53 \pm 0.14$ & $0.49 \pm 0.24$ & $0.04 \pm 0.10$ & 127 & 0.956 & Insignificant \\
\hline \multicolumn{7}{l}{ Values are expressed as mean \pm Standard Deviation (S.D), $\mathrm{n}=128}$. \\
\hline
\end{tabular}

Table 5 above shows the effects of Cessation and Continuation of the exercise protocol in the mean values of the Inflammatory Cytokines parameters for group 1 and group 2. The table shows an increase with average values of $0.015 \pm 0.01 \mathrm{pg} / \mathrm{ml}$ and $0.021 \pm 1.35 \mathrm{pg} / \mathrm{ml}$ in the TNF- $\alpha$ and IL- 6 respectively and a mean decrease of $0.042 \pm 0.10 \mathrm{pg} / \mathrm{ml}$ in IL-10 following a continuation of the exercise protocol as seen in group 2. This means that cessation of the exercise protocol after 24 days as seen in group 1 produced a lesser effect compared to continuation of the exercise protocols as seen in group 2. However, the changes 
were not statistically significant except with IL-6. The results of this table clearly demonstrates that the effect of duration on the observed changes are not statistically significant.

Table 6

The Comparative Effects of Continuation and Increase in Intensity of the Exercise Protocol at the End of 48days on Inflammatory Cytokines

\begin{tabular}{|c|c|c|c|c|c|c|}
\hline Parameters & $\begin{array}{l}\text { Exercise Group } \\
2\end{array}$ & Exercise Group. & Diff. & Df & $\begin{array}{l}\text { Sig.(2- } \\
\text { tailed) }\end{array}$ & Remark \\
\hline $\begin{array}{l}\text { TNF- } \\
\mathrm{a}(\mathrm{pg} / \mathrm{ml})\end{array}$ & $0.065 \pm 0.026$ & $0.10 \pm 0.11$ & $0.045 \pm 0.07$ & 127 & 0.197 & Insignificant \\
\hline IL-6(pg/ml) & $0.057 \pm 0.014$ & $0.07 \pm 0.28$ & $0.013 \pm 0.35$ & 127 & 0.082 & Insignificant \\
\hline $\begin{array}{l}\text { IL- } \\
10(p g / m l)\end{array}$ & $0.493 \pm 0.24$ & $0.77 \pm 0.3$ & $0.2 \pm 0.06$ & 127 & $0.009 *$ & Significant \\
\hline
\end{tabular}

Table 6 shows the effects of Continuation and Increase in intensity of the exercise protocol in the mean values of the parameters of the Inflammatory Cytokines. Results shows an increase with average values of $0.045 \pm 0.07 \mathrm{pg} / \mathrm{ml}$ and $0.013 \pm 0.35 \mathrm{pg} / \mathrm{ml}$ in the TNF- $\alpha$ and IL-6 respectively and a mean decrease of $0.2 \pm 0.06 \mathrm{pg} / \mathrm{ml}$ in IL-10, following an increase in the intensity of the exercise protocol as seen in group 3 . These values were however, not significant except with IL-10. The results of this table clearly demonstrates that the effect of increase in intensity of the exercise protocols on the observed changes are not statistically significant but significantly boosted the anti-inflammatory effect. 
Table 7

Analyses of variance for the three groups on Inflammatory Cytokine Parameters Inflammatory biomarkers

\begin{tabular}{|c|c|c|c|c|c|c|}
\hline Parameter & \multicolumn{2}{|c|}{ Sum of Squares } & \multicolumn{2}{|r|}{ Mean Square } & \multicolumn{2}{|c|}{ Sig. } \\
\hline \multirow[t]{3}{*}{$\begin{array}{l}\text { TNF } \\
(\mathrm{pg} / \mathrm{ml})\end{array}$} & $\begin{array}{l}\text { Between } \\
\text { Groups }\end{array}$ & .026 & 2 & .013 & 2.420 & 0.100 \\
\hline & Within Groups & .240 & 189 & .005 & & \\
\hline & Total & .266 & 191 & & & \\
\hline \multirow[t]{3}{*}{ IL6 (pg/ml) } & $\begin{array}{l}\text { Between } \\
\text { Groups }\end{array}$ & .010 & 2 & .005 & 11.611 & 0.060 \\
\hline & Within Groups & .020 & 189 & .000 & & \\
\hline & Total & .030 & 191 & & & \\
\hline \multirow[t]{3}{*}{$\begin{array}{l}\text { IL10 } \\
(\mathrm{pg} / \mathrm{ml})\end{array}$} & $\begin{array}{l}\text { Between } \\
\text { Groups }\end{array}$ & .705 & 2 & .353 & 6.167 & 0.054 \\
\hline & Within Groups & 2.574 & 189 & .057 & & \\
\hline & Total & 3.279 & 191 & & & \\
\hline
\end{tabular}

\section{Values are expressed as mean \pm Standard Deviation (S.D), $\mathrm{n}=192 .{ }^{*} \mathrm{P}<0.05$}

Table 7 shows One-way analyses of variance of the inflammatory cytokine mean difference across the three groups. There is no statistical significance following a one way analysis of variance in the three exercise procedures on the values of the inflammatory cytokine parameters. The results of this table clearly demonstrates that Isometric handgrip exercise at 30\% MVC promotes anti-inflammatory effect which is not predominantly affected by the duration and or increase in intensity.

\section{Discussion}

The widespread deleterious health effect of augmented inflammatory state critically calls for identification of therapies that could reduce inflammation and physical exercise is currently becoming a promising panacea to this ubiquitous health burden. The main focus of this study was to assess the responses of selected cytokines to isometric handgrip exercise and identify possible effects of continuation, cessation and increase in intensity of the isometric exercise. The effects of physical activity on systemic inflammation have not been extensively studied and available data have shown controversial reports [2, 3]. The outcome of this investigation shows that the isometric handgrip exercise protocols lead to a significant alteration in the resting values of the inflammatory cytokines post-exercise. Conspicuously, the isometric handgrip exercise induced a decline in the pro-inflammatory cytokines and gave rise to an increase in the anti-inflammatory cytokines. The results further revealed that continuation of the exercise protocol after 24days enhanced and produced a significant decrease of the pro- 
inflammatory cytokines and a rise in anti-inflammatory cytokine. The exercise induced changes in inflammatory cytokine levels have been reported to be related to the type, intensity, and duration of exercise and endurance capacity of the subject [15]. Many studies indicated that acute exercise increased inflammatory cytokines but opposite effects were observed after chronic exercise [9]. Several studies have reported reductions in pro-inflammatory cytokines following physical activities indicating that longterm exercise training may reduce chronic low grade systemic inflammation [15].

The exercise trials in this study resulted in significant changes in the post exercise resting values of the inflammatory cytokines. Notably, there was a reduction in the pro-inflammatory cytokines and an increase in the anti-inflammatory cytokines. The results further revealed that continuation of the exercise protocol after 24days as seen in group 2 resulted in a further reduction of the pro-inflammatory cytokines and an increase in anti-inflammatory cytokine compared to cessation of the exercise protocol after 24days as seen in group 1 though the changes were not statistically significant except with the interleukin 6 . On the other hand, increase in the intensity of the exercise protocol as seen in group 3 significantly further reduced the resting values of interleukin 6 and increased interleukin 10 but the reduction on tumor necrotic factor was not significant compared to continuation of the exercise protocol after 24 days.

Moreover, increase in exercise intensity as seen in group 3 significantly further reduced the resting values of interleukin 6 and tumor necrotic factor but increased interleukin 10. In group 1, there was a mean reduction of $0.05 \pm 0.036 \mathrm{pg} / \mathrm{ml}$ in the tumor necrotic factor (TNF- $\mathrm{a}$ ) and $0.057 \pm 0.014 \mathrm{pg} / \mathrm{ml}$ in interleukin 6 (IL-6) whereas a mean rise of $0.53 \pm 0.14 \mathrm{pg} / \mathrm{ml}$ in interleukin 10 (IL-10) was observed. Similarly, the group 2 participants show a mean reduction of $0.065 \pm 0.026 \mathrm{pg} / \mathrm{ml}$ and $0.057 \pm 0.014 \mathrm{pg} / \mathrm{ml}$ in the tumor necrotic factor (TNF-a) and interleukin 6 (IL-6), respectively and an increase of $0.493 \pm 0.24 \mathrm{pg} / \mathrm{ml}$ in interleukin 10 (IL-10). In group 3, the participants also had a mean reduction of $0.10 \pm 0.11 \mathrm{pg} / \mathrm{ml}$ and $0.07 \pm 0.28 \mathrm{pg} / \mathrm{ml}$ in tumor necrotic factor (TNF-a) and interleukin 6 (IL-6) respectively and an increase of $0.77 \pm 0.32 \mathrm{pg} / \mathrm{ml}$ in interleukin 10 (IL-10). These changes were interestingly statistically significant in all the three exercise groups; thus it appears that the inflammatory cytokines has a high sensitivity to isometric exercise protocols. Despite the seeming controversies surrounding the effect of physical exercises, the potential for regular physical exercise as an anti-inflammatory intervention is increasingly being recognized [4].

Exercises have been applied as a means of intervention in inflammatory disorders even in conditions where it was previously contraindicated [20]. Comparing the effects of cessation and continuation of the exercise protocols shows an increase with average values of $0.015 \pm 0.01 \mathrm{pg} / \mathrm{ml}$ and $0.021 \pm 1.35 \mathrm{pg} / \mathrm{ml}$ in the TNF- $\mathrm{a}$ and IL- 6 respectively and a mean decrease of $0.042 \pm 0.10 \mathrm{pg} / \mathrm{ml}$ in IL-10 following a continuation of the exercise protocol as seen in group 2 showing that duration of the exercise protocol has a direct proportional effect in the values of these parameters. Nevertheless, these differences did not show any statistical significant but IL-6. On the other hand, the comparative effects of Continuation and Increase in intensity of the exercise protocol shows an increase with average values of $0.045 \pm 0.07 \mathrm{pg} / \mathrm{ml}$ and $0.013 \pm 0.35 \mathrm{pg} / \mathrm{ml}$ in the TNF- $\alpha$ and IL-6 respectively and a mean decrease of $0.2 \pm 0.06 \mathrm{pg} / \mathrm{ml}$ in IL- 10 , following an increase in the intensity of the exercise protocol as seen in group 3 . These differences were 
also not statistically significant but IL-10. An analysis of variance (ANOVA) shows no significant difference in the three exercise groups in all the variables analysed.

Empirical data from large sample size and cohort studies have consistently shown an association between physical activity and inflammation. Specifically, lower IL-6 and TNFa has been reported to be associated with greater volumes of physical activity in the health, aging and body composition study [21]. Taaffe et al., reported that IL-6 concentrations was inversely proportional to the number of reported hours of physical activity spent in a year [22]. Reuben et al., also reported low concentrations of IL-6 in elderly persons with peak levels of leisure activity $[23,24]$.

These reports are consistent with the results of this study. However, this study employed an intervention designed with a quantifiable exercise protocol in contrast to the aforementioned reports. Since elevated levels of TNF- $a$ and IL-6 have been linked to morbidity and mortality of conditions such as obesity, insulin resistance, type 2 diabetes, cardiovascular diseases and inflammatory diseases such as rheumatoid arthritis, Cohn's disease, ankylosing spondylitis, and psoriasis $[2,9,19,20,25]$, the reductions observed in the values of these cytokines in this present study would be of much health benefit. The results of this study showed that 24 to 48 consecutive days of isometric handgrip exercise resulted in reductions in the values of pro-inflammatory cytokines (decreased serum levels of TNF- $a$ and IL-6) and elevation of anti-inflammatory cytokine (IL-10) in middle aged prehypertensive individuals.

\section{Conclusion}

Generally, the exercise-induced changes in the levels of the cytokines (TNF- $a$, IL-6 and IL-10) should improve and/or prevent metabolic abnormalities and protect against the occurrence of chronic diseases. The exercise protocols utilized in this study could therefore be replicated as a prophylactic measure and/or as adjunct to other remedies as it could have a direct application. Future studies using the same exercise protocols should focus on monitoring the chronic effect over a longer duration.

\section{Declarations}

The authors wish to declare that the study was conducted without any financial or commercial relationships which may construe as a potential conflict of interest to this article.

Author Contributions: Conceptualization, O.U.G., N.E.K. and I.J.C.; Data curation, O.U.G., N.Z.O., N.E., E.C., E.E, F.U,; Methodology, O.U.G.; Project administration, C.E.; Supervision, N.E.K. and I.J.C.; Validation, A.-C.E.;

Writing-original draft, O.U.G. and C.O, Writing-review \& editing, O.U.G.

All authors have read and agreed to the published version of the manuscript.

Funding: This research received no external funding. 
Institutional Review Board Statement: The study was conducted according to the guidelines of the Declaration of Helsinki and jointly received institutional ethical approval of Federal Medical Centre Asaba, Delta State (FMC/ASB/A81.VOLXII/101) and Faculty of Basic Medical Sciences, Delta State University, Abraka, Delta State (REC/FBMS/DELSU/18/16/103).

Informed Consent Statement: Informed consent was obtained from all subjects involved in the study.

Data Availability Statement: Not applicable.

Conflicts of Interest: The authors wish to declare that there are no potential conflicts of interest to this article.

\section{References}

1. Monastero, R.N. and Pentyala S. Cytokines as Biomarkers and Their Respective Clinical Cutoff Levels: International Journal of Inflammation 2017:1-11

2. Danlu, Wu, Trinh, L.D., Bruce, P.B. and David, R.W. Long-Term Measurements of Human Inflammatory Cytokines Reveal Complex Baseline Variations between Individuals: The American Journal of Pathology, 2017: 187(12):2620-2626

3. Moon, M.K., Cho, BJ, Lee, Y.J., Choi, S.H., Lim, S., Park, K.S., Park, P., and Jang, C. The effects of chronic exercise on the inflammatory cytokines interleukin- 6 and 2 tumor necrosis factor- $a$ are different with age. Applied Physiology, Nutrition, and Metabolism. 2012: 37(4):631-636

4. Beavers, K.M., Brinkley, T.E. and Nicklas, B.J. Effect of exercise training on chronic inflammation. Clin Chim Acta. 2010; 411(11): 785-793.

5. Megan, C. Chronic Disease Prevention and Management: National Conference of State Legislatures, 2013; 42: 2-7.

6. Khan, M. and Mensah G. Changing practices to improve dietary outcomes and reduce cardiovascular risk: A food company's perspective. Purchase, NY: Background Paper Commissioned by the Committee; 2009.

7. Centers for Disease Control and Prevention. The Power of Prevention: Chronic Disease. The Public Health Challenge of the 21st Century 2009.

8. Santos, R.V., Viana, V.A., Boscolo, R., A., Marques, V., G., Santana, M., G. and Lira, F., S. Moderate exercise training modulates cytokine profile and sleep in elderly people. Cytokine, 2012: 60:731-735.

9. Windsor, M.T., Bailey, T.G., Perissiou, M., Meital L., Golledge, J., Russell, F.D. and Askew, C.D. Cytokine Responses to Acute Exercise in Healthy Older Adults the Effect of Cardiorespiratory Fitness: Frontiers in Physiology. 2018; 9:203.

10. Petersen, A.M., and Pedersen, B.K. The anti-inflammatory effect of exercise. J. Appl. Physiol. 2005; 98:1154-1162.

11. Suzuki, K. Cytokine Response to Exercise and Its Modulation: Antioxidants; 2017: 7(1): 1717. 
12. Hung, Y.L. and Suzuki, K. The pattern recognition receptors and lipopolysaccharides (LPS)-induced systemic inflammation. Int. J. Res. Stud. Med. Health Sci., 2017; 2:1-7.

13. Lim, C.L. and Suzuki, K. Systemic inflammation mediates the effects of endotoxemia in the mechanisms of heat stroke. Biol. Med. 2017; 9: 376.

14. Petersen, A.M., and Pedersen, B.K. The role of IL- 6 in mediating the anti-inflammatory effects of exercise. J. Physiol. Pharmacol. 2006; 57(10), 43-51.

15. Koh, Y. and Park, S. Responses of inflammatory cytokines following moderate intensity walking exercise in overweight or obese individuals; Journal of Exercise Rehabilitation, 2017; 13(4):472-476.

16. Troiano, R.P., Berrigan, D., Dodd, K.W., Masse, L.C., Tilert, T., and McDowell, M. Physical activity in the United States measured by accelerometer. Med. Sci. Sports, 2008; 40:181-8.

17. Chrysant, SG. Current Evidence on the Hemodynamic and Blood Pressure Effect of Isometric Exercise in Normotensive and Hypertensive Persons. J. Clin. Hypertens (Greenwich), 2010; 12:721-726.w4

18. Fernando, O.C. and Denise, V.M. Physical exercise; inflammatory process and adaptive condition: an overview: Rev Bras Cineantropom Desempenho Hum, 2011; 13(4):320-328.

19. Holbrook, J., Lara-Reyna, S., Jarosz-Griffiths, H. and McDermott, M.F. Tumor necrosis factor signaling in health and disease: Research, 2018; 8:111.

20. Salamat, K.M., Azarbayjani, M.A., Yusof, A. andDehghan, F. The response of pre-inflammatory cytokines factors to different exercises (endurance, resistance, concurrent) in overweight men: Alexandria Journal of Medicine, 2016; 52:367-370.

21. Colbert, L.H., Visser, M., Simonsick, E.M. Physical activity, exercise, and inflammatory markers in older adults: findings from the Health, Aging and Body Composition Study. J Am Geriatr Soc., 2004; 52:1098-1104.

22. Taaffe, D.R., Harris, T.B., Ferrucci, L., Rowe, J., and Seeman, T.E. Cross-sectional and prospective relationships of interleukin-6 and C-reactive protein with physical performance in elderly persons: MacArthur studies of successful aging. J Gerontol A Biol Sci Med Sci., 2000; 55: 709-715.

23. Reuben, D.B., Judd-Hamilton, L., Harris, T.B., Seeman, T.E. The associations between physical activity and inflammatory markers in high-functioning older persons: MacArthur Studies of Successful Aging. J Am Geriatr Soc. 2003; 51:1125-1130.

24. Danlu, Wu, Trinh, L.D., Bruce, P.B. and David, R.W. Long-Term Measurements of Human Inflammatory Cytokines Reveal Complex Baseline Variations between Individuals: The American Journal of Pathology, 2017; 187, No. 12.

25. Mendham, A.E., Duffield, R., Marino, F., and Coutts, A.J. Differences in the acute inflammatory and glucose regulatory responses between small sided games and cycling in sedentary, middle-aged men. J. Sci. Med. Sport, 2015; 18:714-719.

\section{Figures}




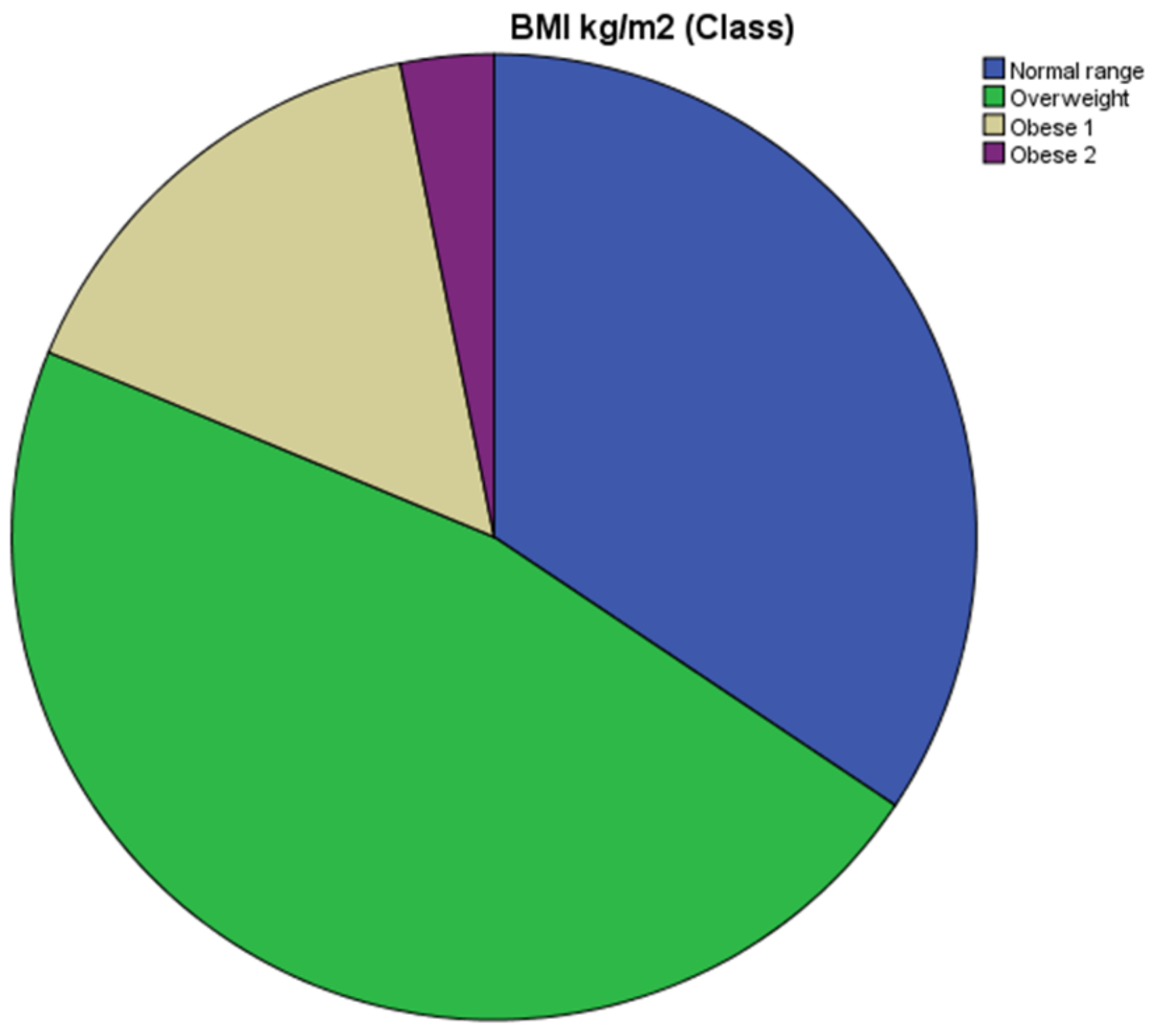

Figure 1

Classification based on body mass index (BMI Classification) 


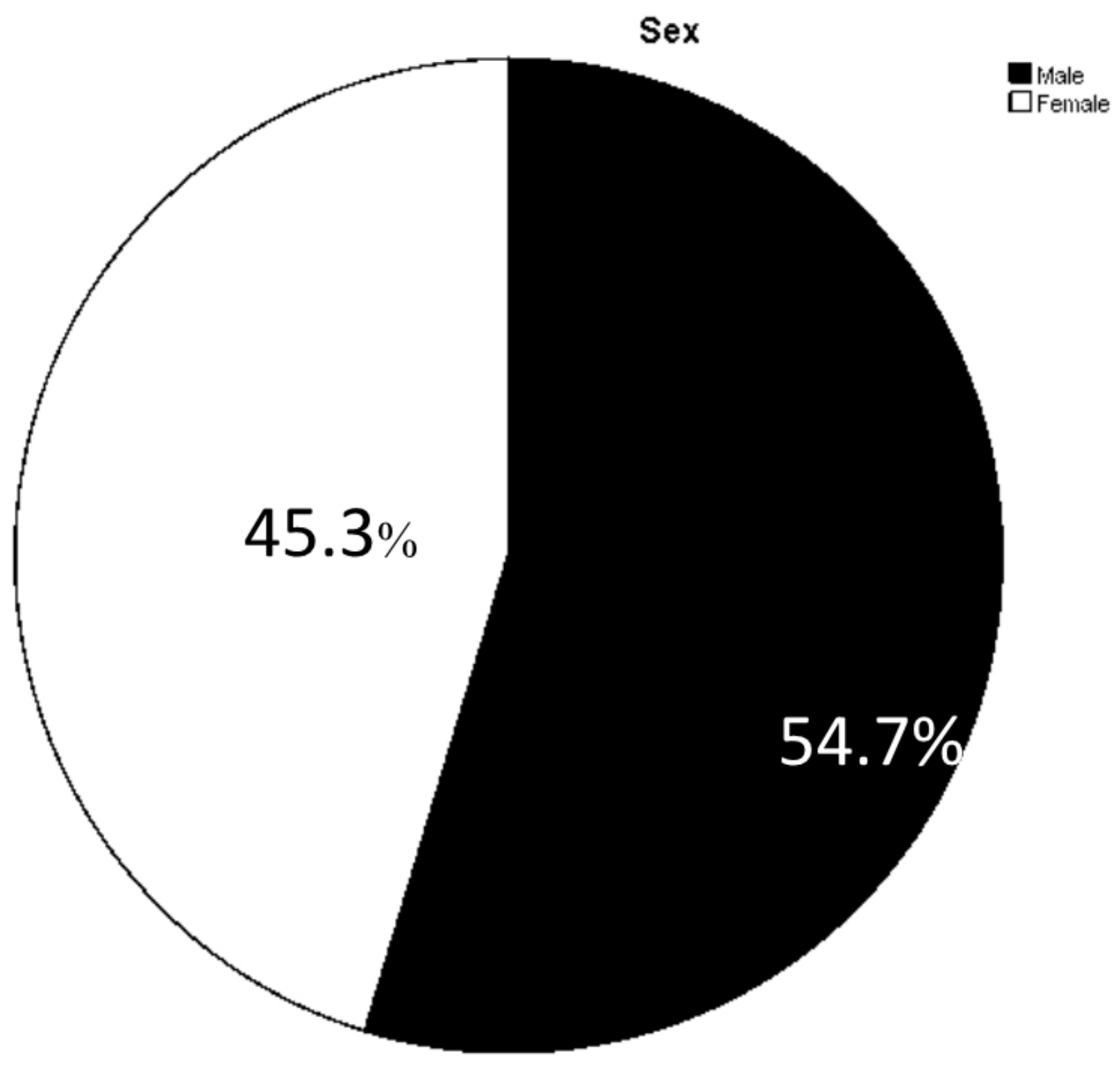

Figure 2

Classification of the participants based on sex 